\title{
Far from Deficient, close to Perfect: Ambulatory Surgery Centers are the Perfect Place for Outpatient Surgery
}

\author{
Awad Magbri MD*, Suhail Aftab MD, Vincent Toma MD, Karl Luketic MD, Anita Moses, \\ Karen Miller MSC RN \\ Surgery and Vascular Center at Regency Park, Toledo, $\mathrm{OH}$
}

*Corresponding Author: Awad Magbri, Surgery and Vascular Center at Regency Park, Toledo, OH, Email: elmagbri@hotmail.com

Keywords: Ambulatory surgical centers, Medicare and Medicaid beneficiaries, federal government mandates, accreditation bodies.

\section{EDITORIAL}

The United States struggles with how to deliver a quality health services to its citizens without anchoring a huge economic fall-down. This impediment leads to the emergence of Ambulatory surgical centers (ASCs) as successful transformation model in health care delivery.

Thirty years ago, virtually all surgical procedures are carried out in hospitals. Waits for weeks and months for a surgical procedure are not uncommon, and patients spent several weeks at hospital and several weeks out of these facilities to recover. This healthcare system delivery is inconvenient, inefficient and resented by patients and doctors alike. It costs the United States a great deal of economic resource spending that can be smartly used for other purposes. In many countries, this is still the way to deliver health services, but not any more in the United States.

Since the inceptions of the Ambulatory Surgical Centers in the 1970, a high quality, cost-effective alternatives have emerged as substitutes to the hospital inpatient and outpatient facilities. Physicians faced with frustrations like scheduling delays, limited operating room availability, and challenges in obtaining new state of the art equipment that have been curtailed by hospital budgets and policies, motivated them to provide the impetus for the development of new ASCs. Now physicians have gain direct control over their surgical practices (1). In the ASC setting, physicians are able to schedule procedures more conveniently, assemble teams of specially-trained and highly skilled staff ensures the equipment and the supplies used for these procedures are well suited to their techniques, and designs facilities customized to their skills. For the first time, physicians have found professional autonomy over their work environment and over the quality of care they provide to their patients.

Given the frustrations of physicians from the hospital environment, it is not surprising that physicians continue to have ownership in virtually all $(90 \%)$ of ASCs. Now more and more independent physicians embrace the value of the ASC model (2).

The impact of aging population has put more demand on the increase in surgical procedures and the attendant need for surgical subspecialties. The growth in aging population would be a major force in driving significant demand for surgical services. The forecasted growth in 2020, predicted that $14-47 \%$ of the population would need surgical procedure depending on the specialty (3). Meeting these needs will be a challenge and the solutions include increasing the workload of surgeons in the workforce and improving the efficiency of surgeons. These objectives can effectively be met in the ASCs. The enactment of ASCs can help mitigate the impact of the aging population on the anticipated shortages of the surgical workforces.

The safety and quality of care provided in the ASCs is evaluated by independent observers through three prongs; state licensure, voluntary accreditation, and Medicare certification. The state requires a rigorous initial and ongoing inspection and reporting to the Sates in order to ascertain licensure for the ASC to operate. Annual or biennial inspection is mandatory by 
the State to grant license to ASC. All ASCs serving Medicare beneficiaries must be certified by the Medicare program. The ASC must comply and meet the standards developed by the federal government to ensure the safety of patients and the quality of the services rendered to the beneficiaries. The quality of the facility, the standards of physicians, staff, ancillary services and management must meet the guidelines put by the federal government to standardize the ASCs.

Almost all ASCs choose to go through voluntary accreditation by independent accreditation bodies. The Joint Commission on Accreditation of Healthcare Organization (JCAHO), Accreditation Association of Ambulatory Health Care (AAAHC), and The American Association for the Accreditation of Ambulatory Surgery Facilities (AAAASF) are examples of independent accreditation bodies that requires ASCs to engage in external benchmarking, which allows the ASC to compare its performance to other ASCs facilities.

The Medicare program also limits the scope of surgical procedures reimbursed in ASCs (4). Generally, services provided by the ASCs are limited to elective procedures with short anesthesia and operating time not requiring overnight stay (5). The federal government put regulation for ASCs to provide services specifically designed for selective outpatient surgical services (6).

ASCs consistently, perform better than hospitals when quality and safety is examined. In a recent study the rate of inpatient hospital admission and death in elderly patients following common outpatient surgical procedures are compared between hospitals outpatient's vs ASCs and found that death was lower in freestanding ASCs. ASCs also have demonstrated lower adverse outcome (7).

In our ASC, we provide the following services to our patients;

- Ear nose and throat procedures;

- Removal of tonsils and adenoids, placement of ear tubes, sinus surgeries with navigation, evaluation of upper airways, etc...

- Vascular access maintenance procedures for end-stage kidney disease patients;

- Placement, removal and exchange of tunneled central catheters, angiogram and angioplasties of arterio-venous fistula and grafts, thrombectomies of AVFs and AVGs, balloon assisted maturation of AVFs, stent placement in AVFs and AVGs, obliterations of accessory veins, etc...

- Genito-urinary tract procedures;

- Bladder surgeries, prostate surgeries, removal of kidney stones using cystoscopy with LASER and ESWL (lithotripsy), vasectomies.

- Ophthalmological procedures;

- Cataract removal and lens implants, blepharoplasty,

- Reconstructive plastic surgical procedures;

- Cosmetic and reconstructive surgeries, skin cancer removals and constructive surgeries,

- Podiatry;

- Hammer toes surgeries, bunionectomies, Achilles tendon repair, etc...

Last year, we have achieved the benchmarks of patient satisfactions and the number of procedures performed in our facility. We have performed a total of 4801 procedures. The complication rate is $0.20 \%$, The infection rate is $0.02 \%$. Patient satisfaction is $>93 \%$.

The excellent outcomes associated with the ASCs when safety and quality of services are concerned have made ASCs more successful. It is imperative that the ASCs should push to achieve the excellent outcome and promote this industry to be known to the public through ASC Quality Collaboration.

Recent surveys show that average patient satisfaction in ASCs exceeds $90 \%$ (8). Our patient satisfaction was $>93 \%$ surpassing the reported statistics. Safe and high-quality services, ease of scheduling, greater personal attention and lower costs are among the reasons that make ASCs more productive. Lower costs of coinsurance pay for procedures performed in the ASC along with the patient satisfaction made ASCs more popular in the Medicare payment programs (9). ASCs have also succeeded in curtailing the high-pitched healthcare expenditures that would have never happened had the ASC options were not existed (10). The private insurance companies are also encountered greater saving when they utilize the ACS model.

In conclusion, ASCs have consistently delivered, high quality outcome at a significant savings. As the number of surgical procedures 
performed in the ASCs grows, the Medicare program would certainly make a substantial savings if they embraced the role of ASCs in healthcare delivery. This is also applied to the Medicare beneficiaries who would realize additional out-of-pocket saving (10). This success narrative of ASCs should be circulated in the media and delivered to the vanguard of public perception. The Federal government officials are also seriously obligated to look at this option and adapt policies recommending ASCs.

\section{REFERENCES}

[1] "Ambulatory Surgical Centers" Encyclopedia of Surgery. Ed. Anthony J. Senagore, Thomson Gale, 2004.

[2] Ambulatory surgery centers; a positive trend in health care. www.ascassociation.org/CONNEC T/Other Communities.
[3] Etzioni DA, Liu JH, Maggard MA, Ko CY. The aging population and its impact on the surgery workforce. Ann Surg. 2003; 238(2): 170-7.

[4] Centers for Medicare and Medicaid Services ASC Website, http://www.cms.hhs.gov/center/ asc.asp.

[5] 70 Fed. Reg.pp.68916-68964, November 10, 2005.

[6] 42 C.F.R $\S 416$.

[7] Fleisher LA, Pasternak LR, Herbert R, Anderson GF. Inpatient hospital admission and death after outpatient surgery in elderly patients: importance of patient and system characteristics and location of care. Arch Surg. 2004; 139(1): 67-72.

[8] Press Ganey Associates, 2004.

[9] Deficit Reduction Act of 2005.

[10] Mes PAC, Report to the congress: Medicare Payment Policy, March 2004.

Citation: Awad Magbri, et al, Far from Deficient, close to Perfect: Ambulatory Surgery Centers are the Perfect Place for Outpatient Surgery. ARC Journal of Neuroscience. 2019; 4(3):26-28. doi:dx.doi.org/10.20 431/2456$057 X 0403004$.

Copyright: () 2019 Authors. This is an open-access article distributed under the terms of the Creative Commons Attribution License, which permits unrestricted use, distribution, and reproduction in any medium, provided the original author and source are credited. 